\title{
OPEN
}

Published online: 25 March 2020

\section{Author Correction: Comparison of spectral and spatial denoising techniques in the context of High Definition FT-IR imaging hyperspectral data}

\section{Paulina Koziol, Magda K. Raczkowska, Justyna Skibinska, Sławka Urbaniak-Wasik, Czesława Paluszkiewicz, Wojciech Kwiatek (1) \& Tomasz P. Wrobel}

Correction to: Scientific Reports https://doi.org/10.1038/s41598-018-32713-7, published online 25 September 2018

This Article contains errors due to a mistake made in one of the Matlab scripts written for Signal Distortion (SD) parameter calculations, resulting in erroneous values of SD for PCA and MNF denoising methods.

As a result, in the Results and Discussion section, under the subheading 'Spectral Denoising',

"For HD data both PCA and MNF outclass every other method with the SNR gain in the range of 60 to 240 times, however, this comes at a cost of larger SD values in the 3-12\% range. Moreover, the choice of optimal parameters is not obvious, since the more PCA or MNF bands are used for reconstruction the less signal is lost but the SNR Gain drops (Supplementary Materials S3-S4)."

should read:

"For HD data both PCA and MNF outclass every other method with the SNR gain in the range of 60 to 240 . This also comes with a very little cost of SD values in the $0.5-1.6 \%$ range (comparable to other techniques). Although, the choice of optimal parameters is not obvious, since the more PCA or MNF bands are used for reconstruction the less signal is lost but the SNR Gain drops (Supplementary Materials S3-S4)."

"PCA performs in a similar fashion as in HD, however, MNF has a significantly higher SD, which is almost constant at around $10 \%$, regardless of the noise level."

should read:

"PCA performs in a similar fashion as in HD, however, MNF has a significantly higher SD, which is around 6.7$8.3 \%$, and vary slightly with the noise level."

The correct Fig. 2 appears below as Fig. 1. 


\section{Spectral denoising}

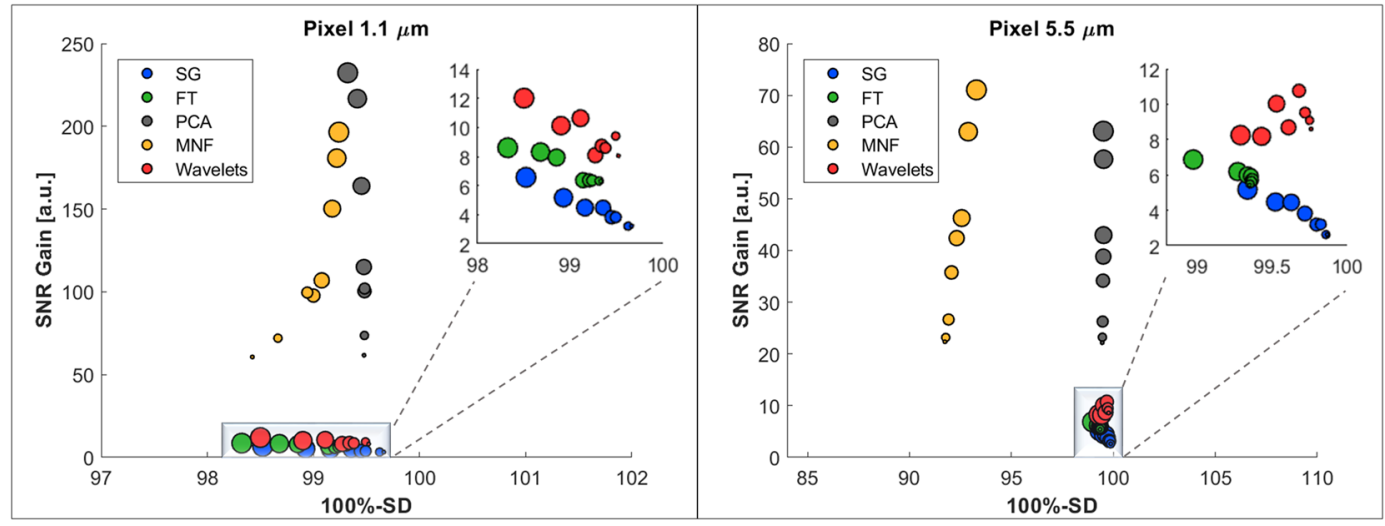

Figure 1. A comparison of spectral denoising techniques as measured by SNR gain (normalized to SNR of the input noisy data) and SD (presented as 100\%-SD) for two different pixel sizes representing HD and standard definition imaging magnifications. Five methods were tested: Savitzky-Golay (SG), Fourier transform (FT), Principal Component Analysis (PCA), Minimum Noise Fraction (MNF) and spectral wavelets (Wavelets). The size of the dots corresponds to the initial noise level, starting with the highest noise for the largest dot (2 scans) and going down to the smallest (256 scans). The optimal parameters for each of the methods and noise levels are given in Supplementary Materials.

Furthermore, in Table 1, in the MNF Spectral denoising technique Cons,

"Difficult algorithm, hard to implement, time and memory consuming computations"

should read:

"Difficult algorithm, very hard to implement, time and memory consuming computations, signal distortion level dependent on the data structure"

Finally, the correct Supplementary Figures S3 and S4 appear below as Figs. 2 and 3 respectively. 


\section{Spectral denoising - Pixel $1.1 \mu \mathrm{m}$}
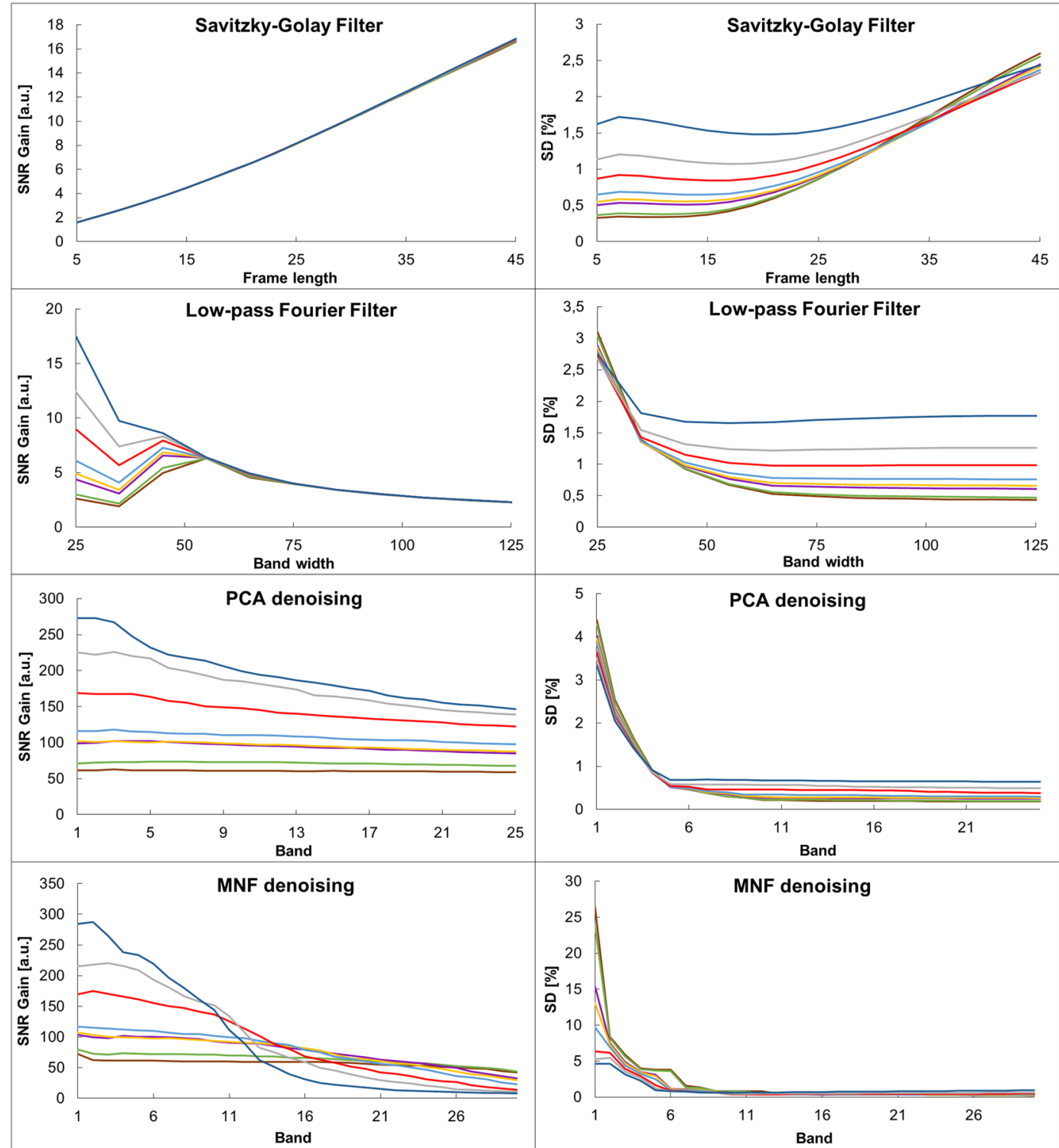

Figure 2. Spectral denoising parameters optimization for PCA, MNF, Low-Pass Fourier Filter and SavitzkyGolay Filter for projected pixel size of $1.1 \mu \mathrm{m}$ and different noise levels in the range from 2 to 256 scans (legend in figure S1) with left column showing SNR Gain and SD on the corresponding graph in the right column. 


\section{Spectral denoising - Pixel $5.5 \mu \mathrm{m}$}
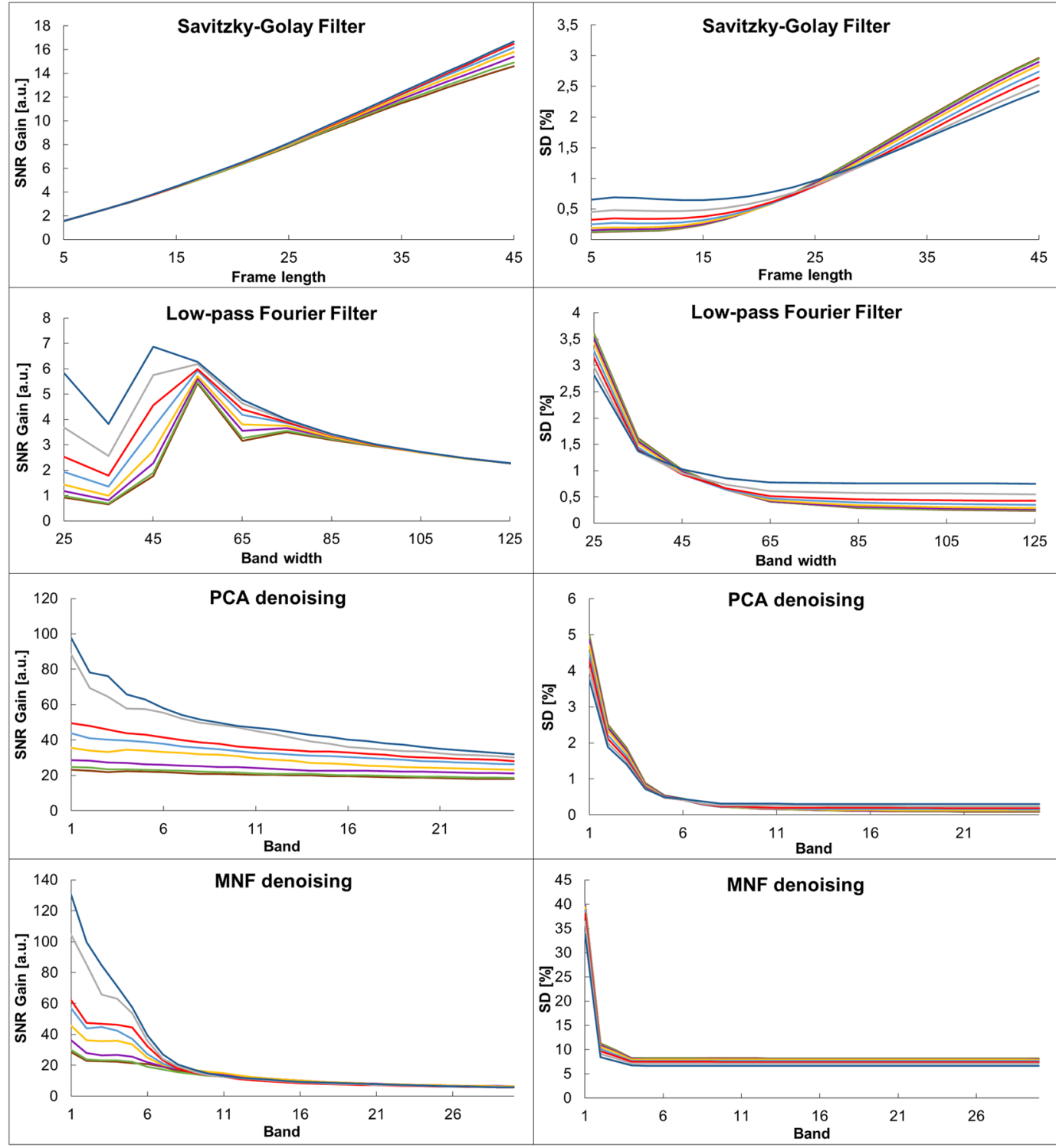

Figure 3. Spectral denoising parameters optimization for PCA, MNF, Low-pass Fourier Filter and SavitzkyGolay Filter for projected pixel size of $5.5 \mu \mathrm{m}$ and different noise levels in the range from 2 to 256 scans (legend in figure S2) with left column showing SNR Gain and SD on the corresponding graph in the right column.

Open Access This article is licensed under a Creative Commons Attribution 4.0 International License, which permits use, sharing, adaptation, distribution and reproduction in any medium or format, as long as you give appropriate credit to the original author(s) and the source, provide a link to the Creative Commons license, and indicate if changes were made. The images or other third party material in this article are included in the article's Creative Commons license, unless indicated otherwise in a credit line to the material. If material is not included in the article's Creative Commons license and your intended use is not permitted by statutory regulation or exceeds the permitted use, you will need to obtain permission directly from the copyright holder. To view a copy of this license, visit http://creativecommons.org/licenses/by/4.0/.

(C) The Author(s) 2020 